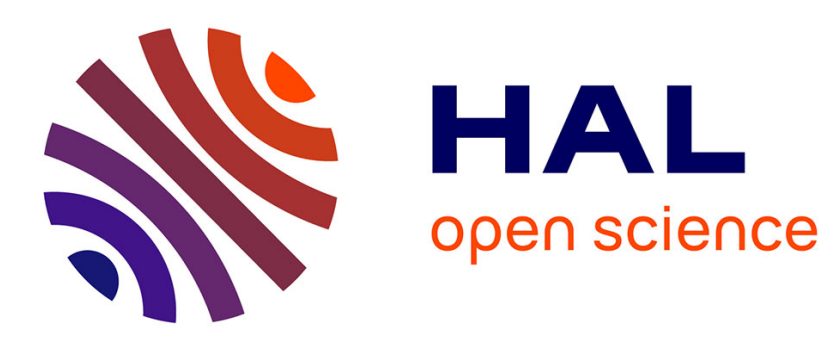

\title{
Spirantisation des obstruantes médiales et formation du système consonantique du vietnamien
}

\author{
Michel Ferlus
}

\section{To cite this version:}

Michel Ferlus. Spirantisation des obstruantes médiales et formation du système consonantique du vietnamien. Cahiers de linguistique - Asie Orientale, 1982, 11 (1), pp.83-106. halshs-01063845v2

\section{HAL Id: halshs-01063845 \\ https://shs.hal.science/halshs-01063845v2}

Submitted on 9 Jun 2015

HAL is a multi-disciplinary open access archive for the deposit and dissemination of scientific research documents, whether they are published or not. The documents may come from teaching and research institutions in France or abroad, or from public or private research centers.
L'archive ouverte pluridisciplinaire HAL, est destinée au dépôt et à la diffusion de documents scientifiques de niveau recherche, publiés ou non, émanant des établissements d'enseignement et de recherche français ou étrangers, des laboratoires publics ou privés. 
Michel Ferlus

\section{SPIRANTISATION DES OBSTRUANTES \\ MÉDIALES ET FORMATION DU SYSTEME \\ CONSONANTIQUE DU VIETNAMIEN $\left.{ }^{*}\right)$}

Version améliorée de l'article de 1982 (remanié d'une communication de 1978). On a corrigé les nombreuses erreurs inhérentes à la nouveauté du sujet et introduit des éléments nouveaux. Le cadre et le style de l'article ont été préservés. Les principales modifications sont en bleu.

Septembre 2014

1. Le viêtnamien est une langue austroasiatique et plus précisément môn-khmer. Des évolutions phonétiques particulières ont éloigné cette langue de celles qui, comme le khmer ou le khamou, sont restées plus conservatrices et donc plus proches du prototype môn-khmer.

Pour comprendre la position du viêt dans l'ensemble des langues môn-khmer, il faut trouver les lois de ses propres changements phonétiques et ces lois nous donneront la clé des correspondances.

Deux exemples vont poser les rapports du viêt et du khamou :

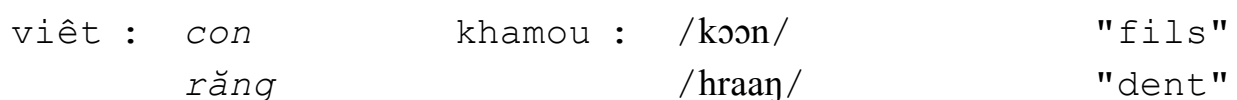

Si pour "fils" la correspondance entre les deux langues est claire et sans ambiguïté, en revanche pour "dent" la ressemblance est trompeuse. răng s'est formé à partir de */ksay/ (encore attesté en thavung) par spirantisation du /s/ médial en $r$ et chute du préfixe /k-/. Quant à /hraay/ il provient de */sraay/. En négligeant la différence de longueur vocalique, encore inexpliquée, on peut remonter à une base verbale */say saay/ sur laquelle le viêt-muong a dérivé le nom de la dent par préfixation de /k-/, tandis que le khamou l'obtenait par infixation de /-r-/. Dans cet exemple, le r du viêt ne correspond pas du tout au /r/ du khamou.

(*) La présente étude est la version remaniée de notre communication "Évolution des obstruantes médiales en vietnamien", à la 2ème Conférence de Linguistique Austroasiatique, Mysore, Inde, 19-21 décembre 1978 . 
2. Actuellement, selon les recherches en cours, on peut postuler en proto viêt-muong (désormais PVM) le système d'initiales suivant :

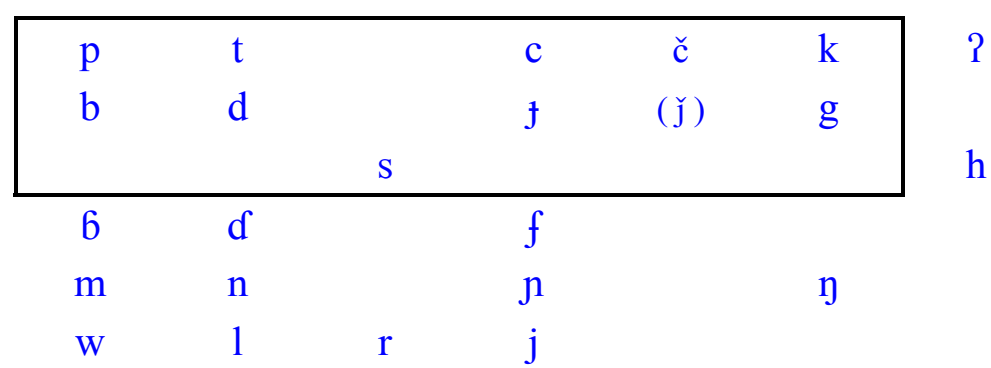

Réorganisation du tableau : On a supprimé les uvulaires */q G/, non reconstructibles, et la sifflante palatale */ś/ (notée /ç/ en API) due à l'influence de la prononciation chinoise, et rajouté la série des préglottalisées $* / 6 \mathrm{~d} f /$, actuellement $m n n h$ en viêt et $/ \mathrm{b} d \mathrm{j} /$ en muong.

Des sonantes sourdes et des occlusives aspirées peuvent être reconstruites à un certain stade historique, mais elles ne remontent probablement pas au PVM.

Seules les unités encadrées seront examinées ici du point de vue de la spirantisation.

3. Pour faciliter la lecture nous donnerons le système actuel du viêtnamien en quốc ngữ avec en regard la phonétique. Les différences dialectales entre le nord N (Hanôi), le centre C (Huê, Da-nang) et le sud S (Sä̈gon) sont d'après L.C. Thompson (1965 : 78-103).

\begin{tabular}{|c|c|c|c|c|c|c|}
\hline$p h / f /$ & th / th $/$ & $x / s /$ & $S$ & $\begin{array}{l}\text { /s/N } \\
/ \mathrm{s} / \mathrm{CS}\end{array}$ & $k h / \chi /$ & $h / \mathrm{h} /$ \\
\hline & $t / \mathrm{t} /$ & $\mathrm{ch} / \mathrm{c} /$ & tr & $\begin{array}{l}/ \mathrm{c} / \mathrm{N} \\
/ \mathrm{t} / \mathrm{CS}\end{array}$ & $\mathrm{k} / \mathrm{c} / \mathrm{q} / \mathrm{k} /$ & \# / $/ 1$ \\
\hline
\end{tabular}

$b / 6 / \quad a / d /$

$\mathrm{m} / \mathrm{m} / \quad \mathrm{n} / \mathrm{n} / \quad \mathrm{nh} / \mathrm{n} / \quad \mathrm{ng} / \mathrm{ngh} / \mathrm{y} /$

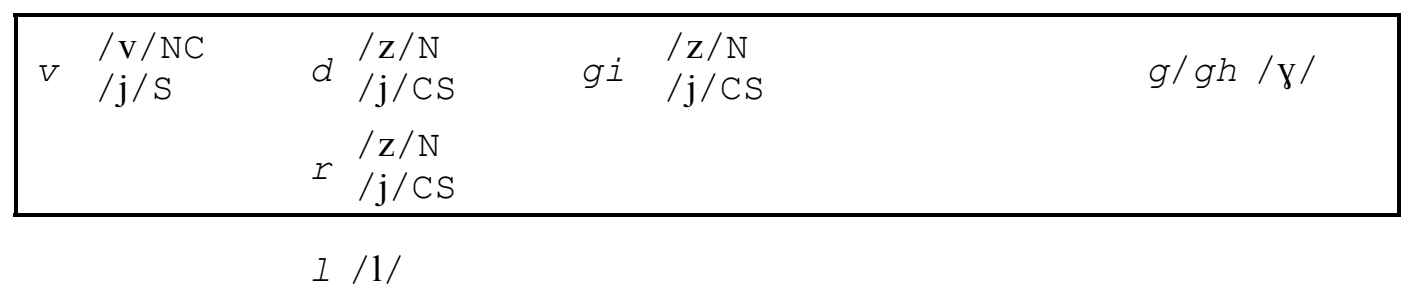

Seules les unités encadrées peuvent être le résultat de la spirantisation des obstruantes médiales du PVM. 
SPIRANTISATION DES OBSTRUANTES MÉDIALES ET

FORMATION DU SYSTEME CONSONANTIQUE DU VIETNAMIEN

\section{LES DOCUMENTS ET LES DONNÉES}

Pour l'histoire du consonantisme viêt nous disposons de quatre catégories de renseignements.

4.1. Les ouvrages anciens :

- Le hua-yi yi-yü (HYYY) dont le vocabulaire chinois-viêtnamien a été étudié pas E. Gaspardonne (1953) et J. Davidson (1975). L'ouvrage est donné du XVIe siècle mais Henri Maspero (1912) pense qu'il a pu être composé à la fin du XVe. La notation du viêt par le chinois n'est ni assez exacte pour assurer toutes les distinctions, ni cohérente en ce sens qu'un même phonème du viêt peut être noté de plusieurs façons en chinois. Le HYYY ne permet pas à lui seul de comprendre les changements du viêt mais il confirme précieusement des faits supposés ou observé par ailleurs. Notons qu'il a dû être élaboré sur le parler de la capitale (Hanoi). Toutefois, des considérations récentes nous enseignent que le parler décrit dans le $H Y Y Y$ n'est pas l'ancêtre direct du vietnamien standard actuel.

Notons l'ouvrage postérieur de Vương Lộc (1995) sur le HYYY (An Nam dịch ngữ).

- Le Dictionarium Annamiticum Lusitanum et Latinum du Père Alexandre de Rhodes (1651), rédigé dans la romanisation qui deviendra le quốc ngư résulte d'une compilation d'ouvrages préexistants et semble élaboré à partir d'un parler du centre.

4.2. Les travaux linguistiques modernes : H. Maspero (1912), A.G. Haudricourt (1954, 1961, 1965), L.C. Thompson (1976), K. Gregerson (1969) et M. Ferlus (1975, 1976).

4.3. Les différents parlers muong cités dans H. Maspero (1912), le muong de khên utilisé par L.C. Thompson (1976) et objet du dictionnaire des Barker (1976). Les parlers archäisants du groupe viêt-muong comme le sách de L. Cadière (1905) ou le thavung de M. Ferlus (1974, 1979).

4.4. Le traitement du sino-viet étudié par H. Maspero (1912) et depuis par de nombreux autres sinologues.

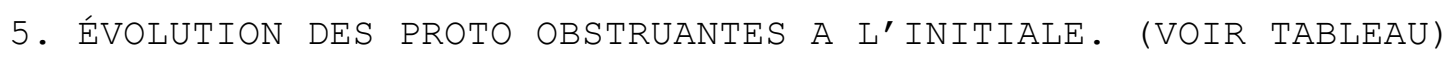

5.1. La perte du voisement des obstruantes est acquise en chinois dès le IXe siècle et semble s'être transmise dans la suite au viêtnamien. La façon dont le sino-viêt a maintenu les distinctions tonales du chinois montre qu'au Xe le viêt avait déjà formé six tons et était donc susceptible d'avoir achevé son dévoisement.

Les six tons du viêt se répartissent en deux séries étymologiques selon la qualité sourde ou sonore des anciennes initiales. Avant les confusions consonantiques il n'y avait que trois tons, ceux qui 
correspondent aux trois inflexions ngang-huyền, sắc-nặng et hỏi-ngã. Les linguistes diachroniciens les désignent par $A B$ et $C$. Ces trois inflexions sont les équivalentes des tons ping, shăng et qù du chinois ancien.

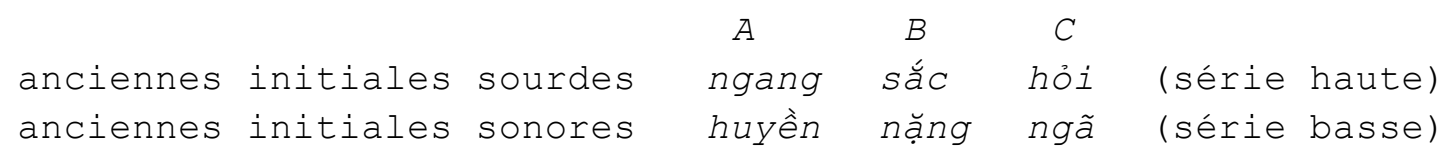

5.2. Les palatales */c $\mathrm{j} /$ et les vélaires */k g/ n'ont subi d'autres changements que le dévoisement. Elles sont notées ch et $\mathrm{k} / \mathrm{c} / \mathrm{q}$.

5.3. Le vietnamien atteste la distinction acquise entre les deux sifflantes */s/ et */ś/ au travers de leur mutation dans les apicales $t / \mathrm{t} /$ et $t$ h $/ \mathrm{th} /$. Rappelons que */s/a a été introduit par l'influence de la prononciation chinoise (thịt "chair, viande", thèm "convoiter"). Voir Nguyễn Tài Cẩn, 1995, p. 85).

Les notations du HYYY montrent que /t/ était déjà formé tandis que /ś/ y semble maintenu. J. Davidson (1975) transcrit les caractères chinois pris comme phonogrammes dans le système Wade-Giles et les mots actuels en th quand ils ne proviennent pas de l'apicale aspirée du chinois, sont transcrits le plus souvent par sh, mais aussi par s/ss, $c h{ }^{e}$ et $c h$.

Je n'ai pas proposé de restituer les sonores correspondantes faute d'argument décisif. Toutefois l'existence en viêt de mots en $t$ ou th avec des tons de la série basse laisse supposer qu'elles ont pu au moins se former à un certain stade historique.

5.4. Les labiales $* / \mathrm{p}$ b/ et les apicales $* / \mathrm{t} d /$ sont actuellement des injectives /6/ et /d/, notées b et $d$. Cette glottalisation, encore inexpliquée, $\mathrm{n}^{\prime}$ a pu que s'effectuer entre le dévoisement et le changement $* / \mathrm{s} />/ \mathrm{t} /$.

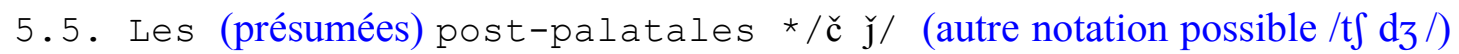
sont aujourd'hui /s/, noté $x$, après un passage probable par /ś/ en viêt moyen. La restitution de la distinction entre sourde et sonore repose sur les séries tonales actuelles. En muong, ces proto phonèmes sont représentés par/s/ ainsi qu'en thavung, semble-t-il.

La reconstruction des post-palatales */č $\breve{j} /$, quoique récente, est justifiée par trois arguments :

- Dans le vocabulaire sino-viêt, $x$ rend l'affriquée palatale aspirée tś du chinois ancien, tandis que les non aspirées tś dź sont rendues par ch.

- En khamou, l'ancienne palatale */c/ du proto môn-khmer a donné /s/ après que */s/ ait donné /h/. Or il existe un /c/ actuel qui correspond pour quelques mots à $x$ du viêt.

$\begin{array}{rlll}\text { proto môn-khmer } & \text { khamou } & \text { viêt } & \\ { }^{\star} \text { s } & \text { hoom } & \text { tom } & \text { "assembler, attacher" } \\ { }^{*} \mathrm{c} & \text { siim } & \text { chim } & \text { "oiseau" } \\ { }_{\star c} & \text { cum } & \text { xum } & \text { "réunir, grouper" }\end{array}$


SPIRANTISATION DES OBSTRUANTES MÉDIALES ET

FORMATION DU SYSTEME CONSONANTIQUE DU VIETNAMIEN

- Dans la transcription chinoise du HYYY l'actuel $x$ est rendu essentiellement par tśch ${ }^{c}$ et $s h$, ce qui semble bien indiquer une prononciation d'affriquée.

- Notons les rapprochements internes suivants :

$\begin{array}{rlll}\text { xay } & \text { "décortiquer, moudre" } & \text { chày, chầy } & \text { "pilon" } \\ \text { xeo } & \text { "soulever au levier, } & \text { chèo } & \text { "rame, ramer" } \\ \text { xum, xúm "se réunir, se grouper" } & \text { chùm } & \text { "grappe, groupe" }\end{array}$

La clé est donnée par "pilon", en sách / kªri/ et en khamou /kndre?/. Cela nous permet de mettre en évidence un ancien infixe */-r-/ à valeur instrumentale. Le muong de khến atteste/khaj/, or dans cette langue /kh/ correspond au viêt $s$ et provient donc de la réduction des groupes d'occlusives avec /r/. Donc les groupes /čr/ se sont réduits puis confondus avec */y/, les tons des dérivés étant de la série basse. Ce changement, propre au vietnamien, s'est produit avant la bipartition tonale et est indépendant de la réduction ultérieure des autres groupes en $s$.

5.6. Il n'y a pas lieu de reconstruire des uvulaires */q G/ contrairement à ce qui a été écrit dans la première version de cette étude. Les arguments avancés étaient bien trop faibles.

5.7. L'occlusive glottale */1/, quoique actuellement assez amuïe, n'a pas subi de changement notoire. Elle n'est pas notée dans l'écriture :

5.8. En muong de khến, compte tenu de la confusion de */s/ et */ś/ en /th/, la série des proto-obstruantes du viêt-muong est représentée par /p th c s k h $\mathrm{p}$. En thavung, les sifflantes se sont confondues avec les post-palatales, nous avons donc actuellement /p t c $\mathrm{s} \mathrm{k} \mathrm{h} \mathrm{P} /$.

\section{6. ÉVOLUTiON DES PROTO-OBSTRUANTES A LA MÉdiALE (VOIR TABLEAU)}

6.1. Par médiale, il faut entendre l'initiale de la deuxième syllabe dans un mot du type présyllabe-syllabe, la présyllabe pouvant être ou non, un préfixe dérivationnel. Ce type syllabique est très fréquent dans les langues môn-khmer. 
Le viêt moderne est de structure monosyllabique mais le viêt ancien d'il y a environ un millénaire était probablement encore dissyllabique comme le sont encore aujourd'hui le sách et le thavung.

6.2. H. Maspero (1912 : 13-39) avait été frappé par les doubles correspondances entre les deux séries en question du viêt d'une part, et les occlusives sourdes d'autre part.

$\begin{array}{lllll}\text { viêt } & \text { b } & \text { d } & c h & \mathrm{k} / \mathrm{c} / \mathrm{q} \\ & \mathrm{v} & \mathrm{d} & \mathrm{gi} & \mathrm{g} / \mathrm{gh} \\ \text { muong } & \mathrm{p} & \mathrm{t} & \mathrm{c} & \mathrm{k}\end{array}$

L'illustre savant avait parfaitement compris que les sonores $v$ d gi g/gh s'étaient formées aux dépens d'occlusives et que, se rencontrant aux deux séries tonales, aussi bien la haute (ngang-sắc-hỏi) que la basse (huyền-nặng-ngã), leur existence ne pouvait être due à une quelconque rétention de l'ancienne série des occlusives.

Il avait en outre remarqué quelques cas assez curieux (1912 :67) où $r$ du viêt correspond à /th/ en muong du nord et à /s/ en muong du sud.

Il est maintenant prouvé, de manière assez sûre, que la série des sonores $V$ d gi $\mathrm{g} / \mathrm{gh} \mathrm{s}^{\prime}$ est formée aux dépens d'occlusives en position médiale de dissyllabe. En examinant les correspondances du viêt et du thavung (Ferlus 1975) on constate une forte corrélation entre les monosyllabes viêt en $v d$ gi $\mathrm{g} / \mathrm{gh}$ et les dissyllabes thavung ayant /p t c k/ à la médiale. D'un autre côté, les monosyllabes viêt en b đa ch $\mathrm{k} / \mathrm{c} / \mathrm{q}$ sont quasi totalement représentés en thavung par des monosyllabes en /p t c k/.

\begin{tabular}{|c|c|}
\hline viêt & thavung \\
\hline $\begin{array}{c}\text { monosyllabes en } \\
v d g i g / g h\end{array}$ & $\begin{array}{l}\text { dissyllabes ayant } \\
/ \mathrm{p} \mathrm{t} \mathrm{c} / \mathrm{a} \text { la médiale }\end{array}$ \\
\hline $\begin{array}{c}\text { monosyllabes en } \\
b \text { đ ch } \mathrm{k} / \mathrm{c} / \mathrm{q}\end{array}$ & $\begin{array}{l}\text { monosyllabes ayant } \\
/ \mathrm{p} \mathrm{t} \mathrm{c} \text { k/ à l'initiale }\end{array}$ \\
\hline
\end{tabular}

Des exemples illustrant ces rapports sont donnés tout au long de cette étude.

De ces rapports entre le viêt et le thavung, j'en avais déduit, sans plus de précision à l'époque, que les sonores $v d$ gi $g / g h$ s'étaient formées par spirantisation d'occlusives médiales qui étaient devenues initiales après la chute des présyllabes. L'explication par la spirantisation avait été étendue à $r$ provenant de $* / s /$. 
A l'époque où j'avais publié ces premiers résultats de mes recherches, je n'avais pas pris en considération le problème des correspondances tonales, faute de pouvoir en donner une explication satisfaisante. A la fin de cette étude, je m'attacherai à montrer que les rapports entre le viêt et le muong de khến ne sont explicables que si la spirantisation s'est produite lorsque la distinction des sourdes et des sonores à l'initiale était encore effective en viêtnamien.

Il convient de signaler que L. Thompson (1976 : 1130-31) a, de son côté, envisagé le phénomène de spirantisation pour expliquer l'évolution du viêt par rapport au muong, toutefois les arguments phonétiques qu'il avance sont différents des nôtres. Conséquemment, l'auteur a été amené à supposer l'existence de dissyllabes en proto viêt-muong et cette idée est d'autant plus méritante qu'il n'avait pas, à ce moment-là, connaissance des données sur le thavung.

Il convient de rappeler que A.G. Haudricourt (1965: 171) avait avancé qu'un préfixe /r-/, attesté dans des mots courants, pouvait être à l'origine de la spirantisation.

6.3. Méthode : Nous avons deux types de preuves à notre disposition pour mettre en évidence une opération de spirantisation.

- Preuves externes, basées sur la comparaison. Viêt: vải thavung: /kpaaś1/ "cotonnade" gấu / $/ \mathrm{cku}^{4} /$ "ours"

- Preuves internes, basées sur les rapprochements entre les termes d'un même complexe dérivationnel. La racine "têter, sucer", viêt bú, anciennement préfixée d'un nominalisateur, probablement */k-/, a donné un dissyllabe qui, par spirantisation et réduction, est aujourd'hui vú "sein". De même chết "mort" a donné giết "tuer" par $l^{\prime}$ action d'un ancien préfixe causatif.

6.4. Nous allons voir comment les correspondances entre les obstruantes du proto viêt-muong et les sources actuelles du viêt vont nous permettre d'en conclure valablement que le phénomène de spirantisation a eu lieu pour six des sept (ou huit, selon le statut reconnu à ś) points d'articulation des proto obstruantes.

Nous utiliserons les données du thavung et du muong de khến. Les tons du thavung sont notés par les chiffres ${ }^{1-3}$ en série haute et ${ }^{2-4}$ en série basse. Les tons du muong de khến sont notés par les signes des tons correspondants en viêt placés après la transcription phonétique, réserve faite que les tons correspondants à nặng-ngã se sont confondus en nặng. Les phonogrammes chinois sont notés dans le système Wade-Giles d'après l'étude de J. Davidson (1975). Le chinois du XVe siècle n'ayant pour sonore que les nasales, les semi-voyelles et la liquide, a noté les spirantes comme les occlusives sourdes.

6.5. */p b/ et $v$.

Aujourd'hui $v$ rend à la fois la spirante issue de */p b/ en médiale et $\mathrm{l}^{\prime}$ ancien */w/. Toutefois dans le Dictionarium la spirante était notée $b$, avec une variante $b \breve{b}$, et était donc distincte de la sonante bilabiale notée $v$ ou $u$. Dans le HYYY, $v<b$ et $b$ sont notés en général par $p$. 


\begin{tabular}{|c|c|c|c|c|c|}
\hline $\begin{array}{l}\text { Viêt } \\
\text { moderne }\end{array}$ & $\begin{array}{c}\text { Dictiona- } \\
\text { rium }\end{array}$ & $H Y Y Y$ & muong & thavung & \\
\hline vua & bua & 380 po & - & - & "roi" \\
\hline vào & cbĕào & 459 pao & paaw & - & "entrer" \\
\hline vải & cbải & 201 pai & paaj $^{2}$ & kpaaś $^{1}$ & "cotonnade" \\
\hline Vôi & bôi & - & pool & $\operatorname{kpuul}^{1}$ & "chaux" \\
\hline vỗ & cbô & 508 pieh & - & $\mathrm{poh}^{1}$ & $\begin{array}{l}\text { "frapper } \\
\text { de la main" }\end{array}$ \\
\hline vàng & uàng & 580 wang & waay' & - & "or" \\
\hline
\end{tabular}

6.6. */t d/ et $d$.

Aujourd'hui d rend à la fois la spirante issue de */t d/ en médiale et $l^{\prime}$ ancien $* / \mathrm{j} /$. Dans le HYYY, $d<* / \mathrm{t} d /$ et đ sont notés le plus souvent par $t$, tandis que $d<* / j / y$ est noté par $y$ ou $j$. Il y avait donc distinction des deux phonèmes selon leur origine alors que dans le Dictionarium, deux siècles plus tard, la confusion est acquise sous $d$ qui, selon la description de A. de Rhodes, devait se prononcer / $/$. On y trouve aussi la graphie dĕ mais elle ne semble être qu'une variante de $a$ tout comme b̆ par rapport à $b$.

La prononciation méridionale / $\mathbf{j} /$ de $d$ (et aussi de gi et $v$ ) ne doit probablement rien à son ancienne valeur et nous serions plutôt en présence d'un aller-retour phonétique.

Il semble que les mots en $d<* / j /$ soient tous d'origine sino-viêt.

$\begin{array}{ccccccc}\text { Viêt } & \begin{array}{c}\text { Dictiona- } \\ \text { moderne }\end{array} & \text { rium } & \text { HYYY } & \text { muong } & \text { thavung } & \\ \text { dê } & \text { dê } & 243 \text { t’e }^{\prime} & \text { te } & - & \text { "mouton" } \\ \text { dưới } & \text { d(ĕ)ưới } & 70 \text { te } & - & - & \text { "murier" } \\ \text { dái } & \text { dĕái } & - & - & \mathrm{ktall}^{3} & \text { "scrotum" } \\ \text { da } & \text { dĕa } & 476 \text { ta } & \text { ta } & - & \text { "peau" } \\ \text { dao } & \text { dĕao } & - & \text { taaw } & - & \text { "couteau" }\end{array}$

mais pour $d<* / \mathrm{j} /$

$\begin{array}{llllll}\text { dần } & \text { dần } & 172 \text { jeng } & - & - & \text { "tigre (cycle)" } \\ \text { dậu } & \text { dậu } & 179 \text { yu } & - & - & \text { "coq (cycle)" } \\ \text { dào } & - & 454 \text { jao } & \text { jaaw } & - & \text { "abondant" } \\ \text { duyên } & - & 582 \text { yen } & - & - & \text { "étain, plomb" }\end{array}$

Cependant, dans un cas, $d$ peut aussi bien se rattacher à l'occlusive apicale viêt-muong qu'à la semi-voyelle sino-viêt. dầu dầu 540 yu $\quad$ - $\quad$ atu $^{1} \quad$ "huile"

$6.7 . * / \mathrm{c} \mathrm{f} /$ et gi.

Le thavung ne nous fournit pas de preuve évidente pour la formation de gi, mais pour giò "patte" le sách nous donne / kco/. On a déjà supposé que giêt "tuer" s'était formé sur chiết "mourir" par un préfixe causatif. Dans le HYYY, gi et ch sont notés en général par ch. 
SPIRANTISATION DES OBSTRUANTES MÉDIALES ET

FORMATION DU SYSTEME CONSONANTIQUE DU VIETNAMIEN

\begin{tabular}{|c|c|c|c|c|}
\hline Viêt moderne & $H Y Y Y$ & muong & thavung & \\
\hline giêng & 123 chieng & cien & - & "premier" \\
\hline giấy & 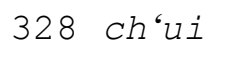 & $c ә j^{\prime}$ & - & "papier" \\
\hline giường & 334 cheng & ci̇̀n & - & "lit" \\
\hline giấm & 540 chen & - & - & "vinaigre" \\
\hline giò & - & $c s^{\prime}$ & kco (sách) & "patte" \\
\hline giấu & - & $\mathrm{cu}^{\prime}$ & - & "cacher" \\
\hline
\end{tabular}

Le viêt moderne, au centre et au sud, confond d et gi en $/ \mathrm{j} / \mathrm{mais}$ cette distinction était assurée au XVIIe comme le prouve le Dictionarium. Au nord $d$ gi et $r$ sont confondus en $/ z /$.

$6.8 . * / \mathrm{k}$ g/ et $\mathrm{g} / \mathrm{gh}$.

C'est la seule unité qui n'ait pas subi de changement notoire depuis sa formation, aujourd'hui elle s'articule entre l'occlusive sonore et la fricative /y/.

Dans le HYYY, g/gh et $\mathrm{k} / \mathrm{c} / \mathrm{q}$ sont en général notés par $k$.

\begin{tabular}{|c|c|c|c|c|c|}
\hline Viêt moderne & HYY & & muong & thavung & \\
\hline gận & 71 & $k^{\prime} e n$ & ken. & tkin ${ }^{1}$ (sách) & "près de" \\
\hline gạo & 219 & kao & kaaw' $^{\prime}$ & $\mathrm{ako}^{3}$ & "paddy" \\
\hline$g u$ & & - & $\mathrm{ku}^{\prime}$ & $\mathrm{cku}^{4}$ & "ours" \\
\hline gừng & 198 & kung & kəəy & ckoon $^{2}$ & "gingembre" \\
\hline gội & & - & kool' $^{\prime}$ & akuul $^{1}$ & $\begin{array}{c}\text { "laver les } \\
\text { cheveux" }\end{array}$ \\
\hline
\end{tabular}

6.9.1.*/s/ et $r$.

Il a été déjà démontré que l'ancien */s/ médial avait, comme les occlusives, subi le phénomène de spirantisation dont l'issue est actuellement représentée par $r$.

Le HYYY donne trois mots écrits avec $r$ en viêt moderne dont on est assez sûr qu'il provient de la fricative sifflante. Deux sont transcrits par ch $j$ et le troisième, "loutre", par $t$ alors qu' on a actuellement rái. On peut penser qu'à cette époque, à Hanoi, le mot était déjà monosyllabique, d'ailleurs des dictionnaires attestent tây, forme rare (ou dialectale ?).

\begin{tabular}{|c|c|c|c|c|c|c|}
\hline & râu & 477 & $\operatorname{ch}^{\prime} \mathrm{u}$ & tho & - & "barbe" \\
\hline & rửa & 495 & jeh & thì $^{?}$ & - & "laver" \\
\hline & răng & & - & than & $\mathrm{ksay}^{1}$ & "dent" \\
\hline mais & rái, tấy & 266 & tai & - & $\mathrm{ps}^{3}$ & "loutre" \\
\hline
\end{tabular}

$D^{\prime}$ autres correspondances entre viêt $r$ et muong / th/ relèvent du même phénomène de spirantisation de l'ancienne sifflante en position médiale. 


$\begin{array}{lll}\text { Viêt } & \text { muong } & \\ \text { ra } & \text { tha } & \text { "sortir" } \\ \text { rá } & \text { tha', } & \text { "corbeille" } \\ \text { rết } & \text { theet' } & \text { "centipède" } \\ \text { rui } & \text { thuuj } & \text { "chevron" } \\ \text { rốn } & \text { thuun', } & \text { "nombril" }\end{array}$

Des paires de mots, de sens identique ou proche, en initiale $t$ et $r$ confirment la spirantisation de $* / \mathrm{s} /$.

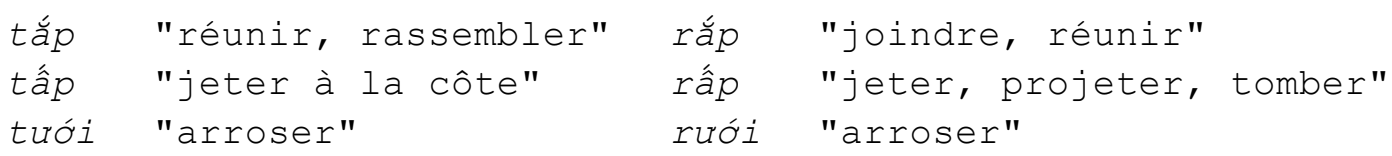

Ces rapprochements n'ont été possibles qu'en utilisant les ressources les plus diverses, en particulier le dictionnaire du P. Gouin (1957).

6.9.2. Note $\operatorname{sur} * /$ ś/ et $r$.

La quasi totalité des mots viêt à initiale th issue de */ś/ sont du sino-vietnamien. Cependant, dans quelques rares mots du stock proprement vietnamien, l'initiale $* / \mathrm{s} /$ a pu être interprétée par */ś/, aujourd'hui th, sous l'influence de la prononciation du chinois. Exemple thịt "chair, viande". Certains rapprochements internes au viêt laissent penser que */ś/ a pu se spirantiser, le résultat étant $r$ comme dans le cas de $* / \mathrm{s} /$. Quoique ténus, ces exemples méritent d'être cités.

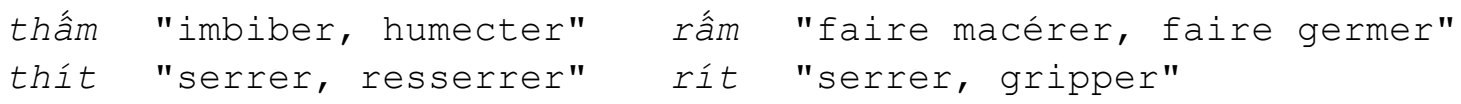

$\begin{array}{ccll}\text { Viêt moderne } & \text { HYYY } & \text { muong } & \\ \text { rau } & 196 \text { shao } & \text { raaw } & \text { "légumes" } \\ \text { rìu } & 362 \text { yu } & - & \text { "hache" } \\ \text { rươu } & 539 \text { jao } & \text { raaw. } & \text { "alcool" } \\ \text { rạng } & 22 \text { yuan } & - & \text { "briller" }\end{array}$

Les transcriptions du HYYY par des phonogrammes chinois ne nous permettent pas de voir si la réalisation de $r<* / s /$ était distincte de celle de $r<* / \mathrm{r} /$, mais il est certain que la prononciation de ce dernier n'était plus celle d'une vibrante, sinon les auteurs du HYYY auraient choisi des phonogrammes en $l$ pour transcrire ces mots. Dans les chữ nôm dont l'élaboration est de plusieurs siècles antérieure à la rédaction du HYYY, ce sont justement des phonogrammes en $l$ qui servent à transcrire les mots viêt en $r$ (H. Maspero 1912). 
SPIRANTISATION DES OBSTRUANTES MÉDIALES ET FORMATION DU SYSTEME CONSONANTIQUE DU VIETNAMIEN

Il faut en outre remarquer que dans le parler qui a servi à élaborer le HYYY, probablement celui de la capitale, les proto phonèmes */r/ et */j/, aujourd'hui notés $r$ et $d$, étaient déjà confondus alors que deux siècles plus tard ils étaient distincts dans le centre comme le montre le Dictionarium. Aujourd'hui encore, la confusion de $r$ et $d$ (et gi) en /z/ est spécifique au nord alors que dans le restant de l'aire vietnamienne la prononciation de $r$, quelle que soit son origine, est celle d'une vibrante distincte des autres phonèmes.

Quand l'usage du quốc ngữ s'est répandu, c'est la prononciation du centre qui a servi de référence et la distinction de $r$ et $d$ était déjà purement graphique pour les locuteurs du nord.

Cette ancienne confusion est la cause de l'alternance graphique entre $r$ et $d$ que $l^{\prime}$ on rencontre pour certains mots.

Certains emprunts au chinois, étymologiquement en $\star / r /$, ont pu se répandre dans l'aire vietnamienne avec la prononciation du nord et sont donc écrits avec $d$. Cela semble être le cas de dậu "(année du) coq" dont l'ancienne initiale est conservée dans le khamou / raw/. En dehors de ces cas particuliers, la vibrante est normalement conservée dans les anciens emprunts au chinois : ràn "enclos", rèm "store", rọc "poulie"...

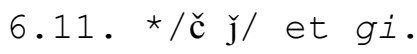

La spirantisation des postpalatales se laisse supposer d'après certains rapprochements à l'intérieur du viêt.

$\begin{array}{ll}\text { xâm "piquer, perforer, } & \text { giâm "bouturer, transplanter" } \\ \text { xum "se réunir" } & \text { gium "aider" } \\ \text { xắc "se quereller" } & \text { giặ "guerre, ennemi, pirate" } \\ \text { xấp "imprégner" } & \text { giấp "arroser" }\end{array}$

Un phénomène de préfixation a dû transformer des racines monosyllabiques en dérivés dissyllabiques et les post-palatales se sont spirantisées en une unité notée aujourd'hui gi et confondue avec celle issue de $\star / \check{c} \breve{j} /$.

6.12. L'occlusive laryngale */1/, non écrite en quốc ngữ, n'a pas subi la spirantisation. 


\section{FORMULATION DE L'HYPOTHESE DES SPIRANTES SOURDES ET SONORES}

7.1. La série des sonores qui s'est formée à la médiale, apparâ̂t dans les deux séries tonales tout comme la série $b$ a ch $x$ $k / c / q$ qui s'est formée à l'initiale.

Il est aujourd'hui bien admis que la répartition en séries dites 'haute' ou 'basse' lors d'une bipartition tonale est conditionnée par la qualité 'sourde' ou 'sonore' de l'unité consonantique précédant la voyelle. Cela nous amène à postuler un stade hypothétique avec opposition de spirante sourde à spirante sonore (stade 2 du tableau).

7.2 S'il y a eu opposition de sourde à sonore pour les spirantes, nous devons admettre que les confusions consonantiques se sont faites au bénéfice des sonores comme le sont actuellement $v$ d r gi g-gh. A l'initiale, comme on l'a déjà vu, la confusion s'est faite au bénéfice des sourdes. Cette différence de comportement est le deuxième fait essentiel, après la spirantisation, qui caractérise les changements phonétiques des unités médiales par rapport aux initiales.

L'examen du thaï (siamois), langue dont l'histoire du phonétisme nous est bien connue depuis les travaux de A.G. Haudricourt (1948), nous fournit un modèle qui va nous permettre de comprendre ce qui a pu se passer en vietnamien.

Le thai a développé une bipartition tonale, son système passant de trois à six tons (puis à cinq par une réduction ultérieure). Les confusions consonantiques se sont faites par dévoisement des occlusives et des fricatives sonores, et par voisement des sonantes sourdes. L'écriture d'origine khméro-indienne, adaptée au thaï vers le XIIIe siècle après une période de tâtonnements, a conservé dans ses formes les valeurs anciennes et confirme ces faits phonétiques d'une manière indiscutable. Les tons sont déterminés en fonction des trois tons primordiaux, des finales, et des séries consonantiques. Ces phénomènes ont été magistralement expliqués par A.G. Haudricourt (1961).

Examinons les labiales à titre d'illustration.

\begin{tabular}{|c|c|c|c|c|}
\hline \multicolumn{5}{|c|}{ Changements des initiales labiales } \\
\hline \multicolumn{2}{|c|}{ proto thai } & \multicolumn{2}{|c|}{ réalisations actuelles } & processus \\
\hline$* \mathrm{ph}$ & $=$ & $\mathrm{ph}$ & ผ & (sourde inchangée) \\
\hline$* p$ & $=$ & $\mathrm{p}$ & ป & (sourde inchangée) \\
\hline$* 6$ & $=$ & $6(>b)$ & บ & (sourde inchangée) \\
\hline$* b$ & $>$ & $\mathrm{ph}$ & พ & dévoisement \\
\hline$*_{f}$ & $=$ & $f$ & ฝ & (sourde inchangée) \\
\hline$*_{\mathrm{V}}$ & $>$ & $\mathrm{f}$ & ฟ & dévoisement \\
\hline *hw & $>$ & $\mathrm{W}$ & หว & revoisement \\
\hline$*_{\mathrm{W}}$ & $=$ & $\mathrm{W}$ & ว & (sonore inchangée) \\
\hline *hm & $>$ & $\mathrm{m}$ & หม & revoisement \\
\hline$*_{\mathrm{m}}$ & $=$ & $\mathrm{m}$ & ม & (sonore inchangée) \\
\hline
\end{tabular}


Dans ce tableau, les sonores */b/ et */v/ s'assourdissent en /ph/ et $/ \mathrm{f} /$, tandis que les sourdes $* / \mathrm{hw} /$ et $* / \mathrm{hm} /$ se revoisent en $/ \mathrm{w} /$ et $/ \mathrm{m} /$. Seule la préglottalisée */b/ semble faire exception car non opposable à d'autres unités. Elle est aujourd'hui pratiquement déglottalisée. Les tons correspondants forment une série dite moyenne dont les unités tendent à se confondre avec ceux des autres séries.

7.3. Sur la foi de ces observations, les consonnes peuvent se répartir en deux catégories selon que les confusions se résolvent en sourdes ou en sonores. Nous aurons d'une part les occlusives et les fricatives, d'autre part les nasales, les liquides et les semivoyelles. Ces deux catégories sont justement celles des OBSTRUANTES et des RÉSONANTES, pour employer deux termes empruntés à la terminologie anglo-saxonne.

Nos spirantes du vietnamien seraient donc à placer dans les résonantes.

7.4. Il faut donc, sur les arguments diachroniques précédents, bien distinguer les fricatives des spirantes, comme cela a été fait implicitement tout au long de cette étude.

Dans l'usage courant, les termes 'fricative' et 'spirante' sont considérés comme synonymes. Cependant, A. Martinet (1956 : 24-25) a écrit :

"Il est indiqué de distinguer entre les articulations relâchées qui tendent vers une articulation de type vocalique, pour lesquelles on réservera le terme de spirantes, et les consonnes d'articulation ferme, nettement caractérisées par le frottement de l'air, qui sont proprement des fricatives".

Dans un article à paraître [publié en 1981], A. Martinet opte définitivement pour une distinction nette entre les deux termes. Il observe, dans les langues les plus diverses, une instabilité générale des spirantes avec une tendance soit à se renforcer en fricatives, soit à s'affaiblir en semi-voyelles ou zéro pour les sonores, ou en spirante laryngale pour les sourdes.

7.5. Le choix des signes de l'Alphabet Phonétique International étant insuffisant pour assurer toutes les distinctions graphiques voulues, A. Martinet suggérait de réserver les caractères romans aux fricatives et les caractères grecs aux spirantes. En ce qui concerne nos besoins en phonétique historique du vietnamien, nous avons / $\phi \beta /$ pour les bilabiales, $/ \theta \delta /$ pour les dentales, et $/ \chi \gamma /$ pour les vélaires. Pour les autres points d'articulation, on a adopté/ç j/ pour les palatales et les post-palatales que rien ne permet de distinguer dans la spirantisation, et enfin /ř ŕ/ pour la sifflante prépalatale, tout en reconnaissant l'aspect théorique des premiers stades du processus de spirantisation. 


\section{LECture du tABLEAu de L'ÉVOLUtion deS SPIRANTES (p. 104)}

Le stade 2 donne l'état hypothétique résultant de la spirantisation des proto obstruantes. La distinction des sourdes et des sonores y est supposée $d^{\prime}$ après les séries tonales actuelles. Le stade 3 indique le nouvel état des spirantes après le voisement des résonantes lors des confusions consonantiques. Remarquons qu'on n'a pas jugé utile d'établir une éventuelle distinction entre des spirantes palatales et post-palatales, les deux étant aujourd'hui représentées par gi. Au stade 4, celui du HYYY, les transcriptions par les phonogrammes chinois, quoique imparfaites, laissent penser qu'il n'y avait que cinq points d'articulation de spirantes, tout comme au stade 5 du Dictionarium et au stade 6 actuel dans la graphie (mais non dans la prononciation).

\section{ACTION DES PRÉSYLLABES SUR LES SPIRANTES}

9.1. Le témoignage du muong est essentiel pour restituer la qualité sourde ou sonore des proto obstruantes médiales, cette langue n'ayant pas subi la spirantisation présente donc un traitement unique.

Dans les mots d'origine monosyllabique, écrits en viêt par b d $t$ th $c h \times \mathrm{k} / \mathrm{c} / \mathrm{q}$, les correspondances tonales entre les deux langues sont régulières de terme à terme.

Dans les mots d'origine dissyllabique, écrits en viêt par $v$ d gi g/gh, les correspondances sont également régulières mais d'inflexion à inflexion, les séries pouvant être différentes. Rappelons qu'il y a lieu de considérer trois inflexions, A (ngang-huyền), B (sắc-nặng) et C (hỏi-ngã) correspondant aux trois tons primordiaux d'avant les confusions consonantiques. Donc, pour les anciens dissyllabes, les correspondances tonales entre le viêt et le muong sont à l'intérieur d'une même inflexion, mais pas forcément de ton à ton. Cela nous conduit à quatre types théoriques de correspondances, dont seulement trois sont possibles.

$\begin{array}{clllll}\text { type } & \text { muong } & \text { viêt } & \text { muong } & \text { viêt } & \\ 1 & \text { haut } & \text { haut } & \text { pool } & \text { vôi } & \text { "chaux" } \\ 2 & \text { haut } & \text { bas } & \text { paaw } & \text { vào } & \text { "entrer" } \\ 3 & \text { bas } & \text { bas } & \text { paaj. } & \text { vại } & \text { "grand vase" } \\ \text { Le type } & 1(\mathrm{~h}-\mathrm{h}) & \text { est de loin le plus } & \text { fréquent, ensuite vient }\end{array}$
$3(\mathrm{~b}-\mathrm{b})$ et plus loin $2(\mathrm{~h}-\mathrm{b})$.

Le muong de khến est parmi l'ensemble des parlers muong un des plus proche génétiquement du viêt, et son témoignage $n^{\prime}$ en est que plus essentiel. Il n'y a pas de différence d'évolution des obstruantes selon que le mot était mono- ou dissyllabique. La répartition du vocabulaire dans les séries tonales s'y est rigoureusement faite selon les lois attendues, haute après les sourdes, basse après les sonores. Sur la foi du muong, il faut dans les exemples ci-dessus restituer une sourde */p/ dans les types 1 et 2 , et une sonore */b/ dans le type 3 . 
9.2. Pourquoi à propos du type 2 (h-b) y a-t-il eu inversion des séries tonales du muong au viêt?

Le fait que cette irrégularité n'apparaisse que dans le vocabulaire d'origine dissyllabique amène à penser qu'il pourrait être dû à la nature de la présyllabe et à son action sur la spirante. Dans le type 2 (h-b), la présyllabe aurait voisé la spirante attendue comme sourde, ceci se serait passé avant la bipartion tonale. Ici, c'est le khmer qui, à l'instar du thä̈, va nous fournir un modèle explicatif pour nous aider à comprendre les développements du vietnamien.

Le khmer a connu les mêmes phénomènes de confusion consonantique, mais tandis que le viêt a conservé les oppositions en doublant le nombre de ses tons, le khmer quant à lui, a (presque) doublé le nombre de ses voyelles par des glissements d'aperture. Sans entrer dans les détails de ces développements assez complexes et variables selon les dialectes, je prendrai quelques exemples avec la voyelle */oo/ (translittérée par o) qui est dans le système, celle qui pose le moins de problèmes diachroniques et dialectaux. Dans les monosyllabes, la voyelle o s'est développée en /ao/ en série haute (après les sourdes), et en /oo/ en série basse (après les sonores). L'examen des dissyllabes est des plus significatifs.

Lorsque la médiale est une occlusive, c'est sa qualité sourde ou sonore qui commande la bipartition vocalique.

série haute: thkol / tkaol/ "regarder vers le bas"
série basse : thgol /tkool/ "émerger"

Lorsque la médiale est une nasale, c'est la qualité sourde ou sonore de la présyllabe qui commande la bipartition vocalique.

$$
\begin{array}{lll}
\text { série haute : } & \text { thnol / tnaol/ "poteau" } \\
\text { série basse : } & \text { jhnok / cyook/ "courber" }
\end{array}
$$

Les spirantes sont des résonantes comme les nasales, et l'on peut penser qu'elles sont, comme elles, susceptibles d'assimiler la qualité sourde ou sonore de la présyllabe.

9.3. En khmer, la situation est relativement simple, la nasale médiale est en harmonie avec la présyllabe, dévoisée comme dans thnol, ou voisée comme dans jhñok, d'où le développement de o en /ao/ ou /oo/. (Notons que le jh de jhriok n'est qu'une graphie de réajustement pour j servant à indiquer une légère aspiration de contact).

En viêt, la situation est plus complexe car nous avons au départ des spirantes sourdes ou sonores selon qu'elles se sont formées à partir d'occlusives sourdes ou sonores. Les trois types de correspondances tonales entre le muong et le viêt nous obligent à supposer des présyllabes sourdes ou sonores combinables avec les spirantes, tant sourdes que sonores. 
Nous allons schématiser ces situations en prenant conventionnellement les signes / $\phi \beta /$ pour symboliser les spirantes sourdes et sonores, et les signes / $k$ a/ pour symboliser les présyllabes agissant comme sourdes ou sonores.

\begin{tabular}{|l|c|c|c|c|}
\hline \multirow{2}{*}{ proto formes } & évolution en vietnamien & séries tonales \\
\cline { 2 - 5 } & bipartition tonale & \\
\hline $1 * k-p$ & $k-\phi=\mathrm{k}-\phi$ & $\phi>\beta$ & $\mathrm{h}$ & $\mathrm{h}$ \\
$2 * a-\mathrm{p}$ & $\mathrm{a}-\phi>\mathrm{a}-\beta$ & $\beta=\beta$ & $\mathrm{h}$ & $\mathrm{b}$ \\
$3 * a-\mathrm{b}$ & $\mathrm{a}-\beta=\mathrm{a}-\beta$ & $\beta=\beta$ & $\mathrm{b}$ & $\mathrm{b}$ \\
\hline
\end{tabular}

(Note : ce raisonnement est valable pour les autres points d'articulation)

Il faut bien insister sur le fait que les signes / $k$ a/ ne sont pas des reconstructions, mais des symboles donnant une idée de l'action de la présyllabe sur la spirante.

/k/ maintient, ou n'influence pas, les spirantes sourdes (type $1: h-h)$.

/a/ revoise les spirantes attendues comme sourdes (type 2 : h-b), et maintient, ou n'influence pas, les spirantes sonores (type 3 : b-b).

On remarque que le cas $* k$-b (type $4: b$-h) impliquant le dévoisement de la spirante sourde / $\beta /$ n'est pas attestée.

Ces développements théoriques ne sont pas une vue de l'esprit, ils s'inspirent des faits observés en thaï, en khmer et aussi dans l'ensemble des langues môn-khmer. D'une manière générale, le dissyllabisme est un des grands problèmes du comparatisme môn-khmer.

Les phonèmes des présyllabes n'évoluent pas comme ceux des syllabes principales. Le mode de jonction inter syllabique est aussi une des grandes sources de troubles dans les correspondances.

\section{ILLUSTRATIONS}

Le thavung $\mathrm{n}^{\prime}$ est cité que pour illustrer les présyllabes, car trop éloigné du viêt et du muong, il est difficilement utilisable pour les correspondances tonales. (les données muong sont d'après Barker, 1976).

\begin{tabular}{|c|c|c|c|c|c|}
\hline type & médiale & muong & viêt & thavung & \\
\hline $1(h-h)$ & $\star / \mathrm{p} /$ & paaj $^{2}$ & Vải & kpaaś $^{1}$ & "cotonnade" \\
\hline & & pool & Vôi & kpuul $^{1}$ & "chaux" \\
\hline & & $\mathrm{pa}^{2}$ & vả & $\operatorname{tpah}^{1}$ & "frapper de la main" \\
\hline & & $\mathrm{paj}^{2}$ & vảy & $\mathrm{kpeh}^{1}$ & "écaille" \\
\hline & & $\mathrm{pu}^{\prime}$ & $v u ́$ & $P u^{3}$ & "sein" \\
\hline
\end{tabular}




\begin{tabular}{|c|c|c|c|c|c|}
\hline & $\star / \mathrm{t} /$ & ta & $d a$ & - & "peau" \\
\hline & & taaw & dao & - & "couteau" \\
\hline & & tiel & dế & - & "criquet" \\
\hline & & tooj' & dối & - & "être faux" \\
\hline & $\star / \mathrm{s} /$ & than' & rắn & $\operatorname{psin}^{3}$ (sách) & "serpent" \\
\hline & & thay & răng & $\mathrm{ksay}^{1}$ & "dent" \\
\hline & & thit ${ }^{?}$ & rưa & - & "laver" \\
\hline & & tho & râu & - & "barbe" \\
\hline & & thooc' & rốt & - & "dernier, fin" \\
\hline & & thaaw' & ráo & - & "sec" \\
\hline & & tha & ra & - & "sortir" \\
\hline & & tha' & rá & - & "corbeille" \\
\hline & & thaat ${ }^{\prime}$ & rát & - & "cuisant, piquant" \\
\hline & & thaj' & ráy & - & "cérumen" \\
\hline & & theet' & rết & - & "centipède" \\
\hline & & thuuj & rui & - & "latte" \\
\hline & & thuuj $^{\text {? }}$ & rủi & - & "malchance" \\
\hline & $\star / \mathrm{c} /$ & $\mathrm{ca}^{\prime}$ & giá & - & "froid" \\
\hline & & caan $^{\prime}$ & gián & - & "blatte" \\
\hline & & $c ə j^{\prime}$ & giấy & - & "papier" \\
\hline & & $\mathrm{cu}^{\prime}$ & giầu & - & "cacher" \\
\hline & & cien’ $^{\prime}$ & giếng & - & "puits" \\
\hline & $\star / \mathrm{k} /$ & kaaj' & gái & pk $\partial^{3}$ & "féminin" \\
\hline & & $\mathrm{kosc}^{\prime}$ & gót & - & "talon" \\
\hline & & kaaj & gai & $\mathrm{tk} \partial^{3}$ & "épine" \\
\hline & & $\mathrm{k} ə ә \mathrm{j}^{?}$ & gửi & - & "envoyer" \\
\hline & & $\mathrm{ku}^{\prime}$ & gấu & $\mathrm{cku}^{4}$ & "ours" \\
\hline $2(h-b)$ & $\star / \mathrm{p} /$ & paaw & vào & - & "entrer" \\
\hline & & posp $^{\prime}$ & $v o p$ & - & "crampe" \\
\hline & $\star / \mathrm{t} /$ & təy' & dựng & - & "construire" \\
\hline & $\star / s /$ & thit ${ }^{?}$ & rũa & - & "se dessécher" \\
\hline & $\star / \mathrm{c} /$ & $\mathrm{ci}$ & $g i$ & - & "interrogatif" \\
\hline & $\star / \mathrm{k} /$ & $\mathrm{ka}$ & gà & $\mathrm{rka}^{1}$ (sách) & "poulet" \\
\hline & & kaaw' $^{\prime}$ & gạo & ako $^{3}$ & "paddy" \\
\hline & & kool' $^{\prime}$ & gội & akuul $^{3}$ & "laver (cheveux)" \\
\hline & & kəəท & gừng & ckoon $^{2}$ & "gingembre" \\
\hline & & $\mathrm{ko}^{?}$ & gõ & akoh $^{1}$ & "frapper" \\
\hline $3(b-b)$ & $\star / \mathrm{b} /$ & piit. & $v i ̣ t$ & - & "canard" \\
\hline & & paj & vày & - & "froisser" \\
\hline & & puun’ & vìng & - & "région, aire" \\
\hline & & puun. & vũng & - & "étang" \\
\hline
\end{tabular}




\begin{tabular}{|c|c|c|c|c|}
\hline \multirow[t]{3}{*}{ */d/ } & taj' & dày & - & "épais" \\
\hline & toon. & dọn & ktoon $^{4}$ & "préparer, arranger" \\
\hline & tuuj' & dùi & - & "marteau" \\
\hline \multirow[t]{3}{*}{ */f/ } & caj’ & giầy & - & "piétiner" \\
\hline & caan` & giàn & - & "tonnelle" \\
\hline & ciəł’’ & giường & - & "lit" \\
\hline \multirow[t]{4}{*}{ */g/ } & kaaj & gài & - & "fixer, attacher" \\
\hline & kən. & gận & tkin ${ }^{3}$ (sách) & "près de" \\
\hline & kət. & gọt & - & "écrémer" \\
\hline & kaak. & gạc & - & "fourche d'arbre" \\
\hline
\end{tabular}

\section{1. ÉVOLUTION DES SPIRANTES}

11.1. Plusieurs fois, au cours de cette étude, nous avons parlé de l'interaction des spirantes sonores et de $* / \mathrm{w} \mathrm{j} \mathrm{r} /$.

Partons d'un système partiel à huit unités, le plus vraisemblablement reconstructible, comprenant les spirantes, les semi-voyelles et la vibrante.

$$
\begin{array}{lllll}
\beta & \delta & \text { r } & j & \gamma \\
w & j & r & &
\end{array}
$$

Très tôt, dans le nord, */r/ $\mathrm{s}^{\prime}$ est confondu avec */j/, probablement sous l'influence du chinois. A l'époque du HYYY, il semblerait que /ŕ/ ait rejoint la confusion, il n'y aurait donc plus que six unités.

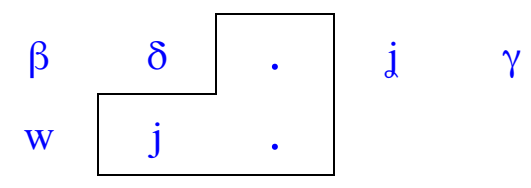

Au XVIIe siècle, dans le centre, le Dictionarium nous montre la confusion de $* / \mathrm{j} /$ avec $* / \delta /$, écrit $d$, et de $* / \hat{r} /$ avec $* / \mathrm{r} /$, écrit $r$. Le système partiel comprend six unités.

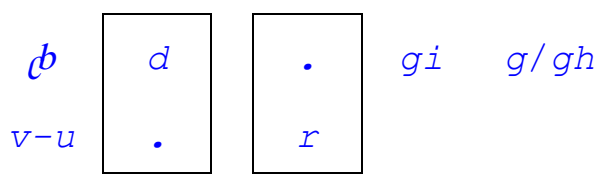

Dans la graphie actuelle, suite à la perte de $b$, nous n'avons plus que cinq unités $v d r g i g / g h$ qui se réduisent à trois ou quatre dans la prononciation selon les régions. 


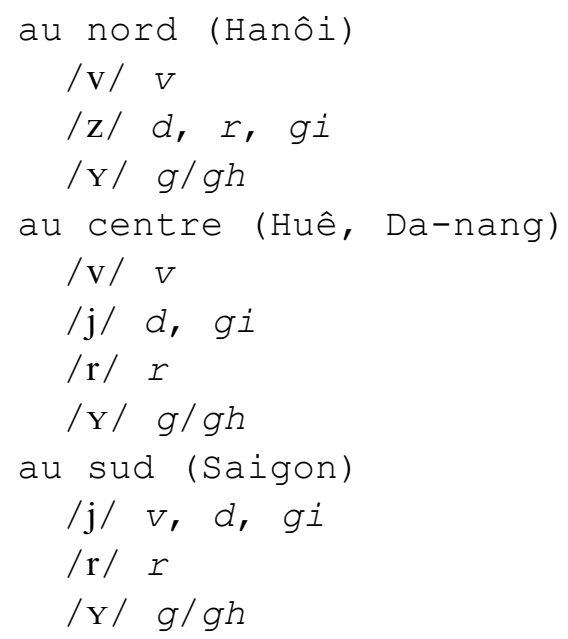

Au sud $v$ est parfois distinct de d gi mais avec une prononciation intermédiaire /bj/ ou /vj/.

11.2. L'évolution des spirantes confirme bien leur caractère instable, et en conséquence leur tendance à la stabilisation par renforcement ou par affaiblissement (rappelons A. Martinet 1981). Dans la plupart des cas, il y a eu renforcement en fricatives sonores comme /V z y/ au nord. Du centre au sud, il y a une importance croissante de l'affaiblissement en /j/ de certaines unités. La pointe sud de l'aire vietnamienne est la plus avancée dans ce sens et, si l'on tient compte d'une certaine palatalisation de g/gh que L. Thompson y a observé (1976 : 1131), on peut se demander si /j/ n'est pas la solution finale vers laquelle tendraient la plupart des spirantes.

\section{LES PRÉGLOTTALISÉES}

A un certain stade historique de l'évolution des parlers viêtmuong il s'est formé une strate de préglottalisées */b d f/ représentées par $m n$ nh en viêt et par /b d j/ en muong de khến.

A côté de ces correspondances régulières, il en existe d'autres plus inattendues, où /b d/ du muong correspondent à $v$ d en viêt.

\begin{tabular}{ccc} 
muong & \multicolumn{2}{c}{ vietnamien } \\
b & (régulier) & (inattendu) \\
d & $m$ & $v$ \\
& $n$ & $d$
\end{tabular}

Celle des bilabiales, la mieux représentée, a retenu l'attention de plusieurs linguistes, M. Barker (1963), E. Hamp (1966), K. Gregerson (1969) et L. Thompson (1976) ; chacun d'eux a proposé une solution. J'ai moi-même pensé que */6 d/ s'étaient spirantisées en $v$ et $d$ parallèlement aux occlusives bilabiales et apicales. 
Un meilleur examen du dictionnaire des Barker (1976) m'amène à remettre tout cela en question. Je pense aujourd'hui que nous avons à faire à des fausses correspondances dues à $d^{\prime}$ anciens emprunts. Le muong de khến en empruntant des mots viêts en $v$ - et $d$ - lorsqu'ils étaient prononcés $/ \beta \delta /$ les aurait identifiés à /b d/. Plusieurs faits permettent de dépister ces emprunts.

- Il y a des mots sino-viêt qui étant en $v d$ ne peuvent provenir que de sonantes.

- On relève des cas de doubles correspondances : vàng "or" est en muong /waay'/ et /baay/, dựng "dresser" est /təy'/ et /dəy./.

- Enfin un mot comme vài "deux, quelques", en muong /baaj/, devrait porter la finale /l/ puisqu'il correspond au khamou /kbaar/.

- Le nombre de ces correspondances inattendues est si élevé pour les bilabiales qu'il est probable que des emprunts au moderne $v$ ont dû être assimilés à /b/ par un phénomène d'hypercorrection. Enfin, ces correspondances sont particulières au muong de khến et ne semblent pas se rencontrer dans les autres parlers muong, d'ailleurs H. Maspero ne les avait pas relevées.

En résumé, les anciennes préglottalisées ne se sont pas spirantisées, et il convient donc de rectifier ce que j'ai écrit sur ce sujet.

\section{SINO-VIETNAMIEN ET ANCIENS EMPRUNTS AU CHINOIS}

On appelle sino-viêt la langue chinoise du Xe siècle, empruntée par les vietnamiens, qui, se trouvant coupée du modèle chinois, a suivi les changements phonétiques propres au viêt.

On trouve du vocabulaire sino-viêt en initiales $v d$ gi. Les deux premières, $v$ et $d$, proviennent des semi-voyelles labiales et palatales, tandis que gi provient du groupe / kj/ du chinois. Ces mots sino-viet en gi ont tous des tons de la série haute quoique l'initiale soit sonore. La semi-voyelle de / $\mathrm{kj} /$ a dû voiser le résultat de la transformation qui s'étant produite après les confusions consonantiques n'avait plus que la spirante palatale dont l'articulation soit assez voisine pour permettre une identification. Il semble qu'un mot proprement viêt-muong ait suivi la même évolution, gió "vent" est / $/ \mathrm{so}^{\prime} /$ en muong et / $\mathrm{kju}{ }^{1} /$ en thavung; de plus, il est attesté avec / kj/ dans plusieurs langues mônkhmer voisines.

Notons que le chinois / khj/ est représenté par $x$.

Il existe une couche d'emprunts chinois, plus ancienne, qui atteste réellement la spirantisation. Le problème est de savoir si le chinois du début de notre ère était dissyllabique, ou bien si le viêt a préfixé des monosyllabes chinois.

\begin{tabular}{|c|c|c|c|}
\hline \multicolumn{2}{|l|}{ viêt } & \multicolumn{2}{|c|}{ sino-viêt } \\
\hline ván & "planche" & bản & "planchette, tablette" \\
\hline lao & "couteau" & đao & "couteau, épée" \\
\hline & "fonte" & cang & "acier" \\
\hline
\end{tabular}




\section{CONCLUSIONS}

L'histoire du vietnamien est celle d'une langue dissyllabique poussée au monosyllabisme sous l'influence du chinois.

Il y a deux procédés pour passer au monosyllabisme :

- Chute de la présyllabe après une période possible de réduction et d'amuïssement. Ce procédé est observable dans la famille des langues môn-khmer.

- Compression du mot en gommant les consonnes médiales. La première manifestation en est la spirantisation qui, si elle se continue normalement, aboutit à une vocalisation.

Le vietnamien a combiné les deux procédés. Les présyllabes sont tombées alors que les spirantes n'avaient pas achevé leur évolution vers un affaiblissement plus poussé ; se retrouvant à l'initiale, elles se sont phonologisées. La survivance de ces spirantes par des sonores a donc un caractère accidentel.

Il est difficile de proposer une date pour la spirantisation, mais il semble qu'elle se soit produite avant les confusions des initiales conduisant aux bipartitions tonales. Le type 2 (h-b) caractérise un stade où une catégorie de présyllabe (Haudricourt avait pensé à /r-/) a dévoisé les initiales sourdes de la syllabe principale. historique.

Tous ces phénomènes font du vietnamien une langue exemplaire pour la phonétique

SUPPLÉMENT : Systèmes tonals du vietnamien, du muong et du thavung

A $\quad$ B $\quad$ C $\quad$ D

\begin{tabular}{|c|c|c|c|c|}
\hline \multirow{2}{*}{ vietnamien } & ngang & sắc & hỏi & sắc \\
\hline & huyền & nặng & $n g \tilde{a}$ & nặng \\
\hline \multirow{2}{*}{ muong } & $\mathrm{a}$ & $a^{\prime}$ & $a^{?}$ & $a t^{\prime}$ \\
\hline & $\mathrm{a}^{\prime}$ & \multicolumn{2}{|c|}{ a. } & at. \\
\hline \multirow{2}{*}{ thavung } & $a^{1}$ & $a^{3}$ & $a h^{1}$ & $a t^{1}$ \\
\hline & $a^{2}$ & $a^{4}$ & $a h^{2}$ & $a t^{2}$ \\
\hline
\end{tabular}


ÉVOLUTION DES OBSTRUANTES MÉDIALES DU PROTO VIET-MUONG AU VIET MODERNE

\begin{tabular}{|c|c|c|c|c|c|c|c|c|}
\hline $\begin{array}{l}\text { Vietnamien } \\
\text { moderne }\end{array}$ & $\begin{array}{l}6 \\
(b)\end{array}$ & $\begin{array}{l}d \\
(d)\end{array}$ & $\begin{array}{l}\mathrm{t} \\
(t)\end{array}$ & $\begin{array}{l}\text { th } \\
\text { (th) }\end{array}$ & $\begin{array}{c}\mathrm{c} \\
(\mathrm{ch})\end{array}$ & $\begin{array}{l}\mathrm{S} \\
(X)\end{array}$ & $\begin{array}{c}\mathrm{k} \\
(k / c / q)\end{array}$ & $\begin{array}{l}? \\
(\#)\end{array}$ \\
\hline $\begin{array}{l}\text { XVIIe (centre) } \\
\text { Dictionarium }\end{array}$ & $\begin{array}{l}6 \\
(b)\end{array}$ & $\begin{array}{l}d \\
(d)\end{array}$ & $\begin{array}{l}\mathrm{t} \\
(t)\end{array}$ & $\begin{array}{l}\text { th } \\
\text { (th) }\end{array}$ & $\begin{array}{c}\mathrm{c} \\
(\mathrm{ch})\end{array}$ & $\begin{array}{l}\text { Ś } \\
(X)\end{array}$ & $\begin{array}{c}\mathrm{k} \\
(k / c / q)\end{array}$ & $\begin{array}{l}? \\
(\#)\end{array}$ \\
\hline $\begin{array}{c}\text { XV-XVIe } \\
\text { (nord) } \\
\text { Hua-yi yi-yü }\end{array}$ & $\begin{array}{l}6 \\
(p)\end{array}$ & $\begin{array}{l}d \\
(t)\end{array}$ & $\begin{array}{l}\mathrm{t} \\
(t)\end{array}$ & $\begin{array}{c}(\hat{S}) \\
(s h / s S \\
\left.c h^{\prime} / c h\right)\end{array}$ & $\begin{array}{c}\mathrm{c} \\
(\mathrm{ch})\end{array}$ & $\begin{array}{c}\check{\mathrm{c}} \\
\left(t s^{6}\right. \\
\left.\operatorname{ch}^{\prime} / s h\right)\end{array}$ & $\begin{array}{l}\mathrm{k} \\
(\mathrm{k})\end{array}$ & $\begin{array}{l}? \\
(\#)\end{array}$ \\
\hline Dévoisement & $\mathrm{p}$ & $\mathrm{t}$ & $\mathrm{s}$ & $\left(\mathrm{S}^{\prime}\right)$ & $\mathrm{c}$ & $\check{c}$ & $\mathrm{k}$ & $?$ \\
\hline
\end{tabular}

\begin{tabular}{|c|c|c|c|c|c|c|c|c|}
\hline $\begin{array}{c}1 \text { Obstruantes } \\
\text { du Proto } \\
\text { viêt-muong }\end{array}$ & $\begin{array}{l}p \\
b\end{array}$ & $\begin{array}{l}\mathrm{t} \\
\mathrm{d}\end{array}$ & $\mathrm{S}$ & (ś) & $\mathfrak{f}$ & $\begin{array}{l}\check{c} \\
(\check{\jmath})\end{array}$ & $\mathrm{g}$ & $?$ \\
\hline
\end{tabular}

\begin{tabular}{|c|c|c|c|c|c|c|c|c|}
\hline 2 Spirantisation & $\begin{array}{l}\phi \\
\beta\end{array}$ & $\begin{array}{l}\theta \\
\delta\end{array}$ & $\begin{array}{l}\check{r} \\
\check{r}\end{array}$ & & & & $\begin{array}{l}\chi \\
\gamma\end{array}$ & \\
\hline 3 Voisement & $\beta$ & $\delta$ & $\mathrm{r}$ & & & & $\gamma$ & \\
\hline $\begin{array}{l}4 \text { XV-XVIe } \\
\text { (nord) } \\
\text { Hua-yi yi-yü }\end{array}$ & $\begin{array}{l}\beta \\
(p)\end{array}$ & $\begin{array}{l}\delta \\
(t)\end{array}$ & $\begin{array}{c}\mathrm{r} \\
\left(s h / c h c^{\prime} / j\right)\end{array}$ & & & & $\begin{array}{c}\gamma \\
(k)\end{array}$ & \\
\hline $\begin{array}{l}5 \text { XVIIe } \\
\text { (centre) } \\
\text { Dictionarium }\end{array}$ & $\begin{array}{c}\beta \\
(b / c \breve{e})\end{array}$ & $\begin{array}{c}\delta \\
(d / d \breve{e})\end{array}$ & $\begin{array}{l}\mathrm{r} \\
(r)\end{array}$ & & & & $\begin{array}{c}\gamma \\
(g / g h)\end{array}$ & \\
\hline $\begin{array}{cc}6 \text { Vietnamien } & \mathrm{C} \\
\text { moderne } & \mathrm{S}\end{array}$ & $\begin{array}{l}\mathrm{v} \\
\mathrm{v} \\
\mathrm{j} \\
(\mathrm{V})\end{array}$ & $\begin{array}{c}\mathrm{z} \\
\mathrm{j} \\
\mathrm{j} \\
(d)\end{array}$ & $\begin{array}{l}\mathrm{Z} \\
\mathrm{r} \\
\mathrm{r} \\
(r)\end{array}$ & & & & $\begin{array}{c}\mathrm{Y} \\
\mathrm{Y} \\
\mathrm{Y} \\
(g / g h)\end{array}$ & $\begin{array}{l}? \\
? \\
? \\
\text { (\#) }\end{array}$ \\
\hline Muong (khến) & $\mathrm{p}$ & $\mathrm{t}$ & th & th & $\mathrm{c}$ & $\mathrm{s}$ & $\mathrm{k}$ & $?$ \\
\hline
\end{tabular}




\section{BIBLIOGRAPHIE}

BARKER, Milton E.

1963 "Proto-Vietnamuong initial labial consonants", Văn-Hóa Nguyệt-San 12 : 491-500.

BARKER, Milton E. et BARKER Muriel A.

1976 Muong-Vietnamese-English Dictionary, Summer institute of Linguistics, microfiches.

CADIERE, P. Léopold

1905 "Les hautes vallées du Sông-gianh", Bulletin de l'Ecole Française d'Extrême-Orient 5 : 347-369.

CUISINIER, Jeanne

1951 Prières accompagnant les rites agraires chez les muong de Mẫn-dức, Publication de l'Ecole Française d'Extrême-Orient, $\mathrm{n}^{\circ} 33$.

DAVIDSON, Jeremy H.C.S.

1975 "A new version of the Chinese-Vietnamese vocabulary of the Ming dynasty", Bulletin of the school of Oriental and African Studies $38(2): 296-315$ et $38(3)$ : 586-608.

DE RHODES, Alexandre

1651 Dictionarium Annamiticum Lusitanum et Latinum, Rome.

FERLUS, Michel

1974 "Problèmes de mutations consonantiques en thavung", Bulletin de la Société de Linguistique de Paris 69(1) : 311-323.

1975 "Vietnamien et proto viêt-muong", Asie du Sud-Est et Monde Insulindien 6(4): 21-55.

1976 "Du nouveau sur la spirantisation ancienne en vietnamien", Bulletin de la Société de Linguistique de Paris 71(1) : 305-312.

1979 "Lexique thavung-français", Cahiers de Linguistique, Asie orientale $5: 71-94$.

GASPARDONE, Emile

1953 "Le lexique annamite des Ming", Journal Asiatique 241 : 354-397.

Gouin, R. P. Eugène

1957 Dictionnaire Vietnamien Chinois Français. Imprimerie d'Extrême-Orient, Saigon.

GREGERSON, Kenneth J.

1969 "A study of middle vietnamese phonology", Bulletin de la Société des Etudes Indochinoises 44(2) : 131-193. 


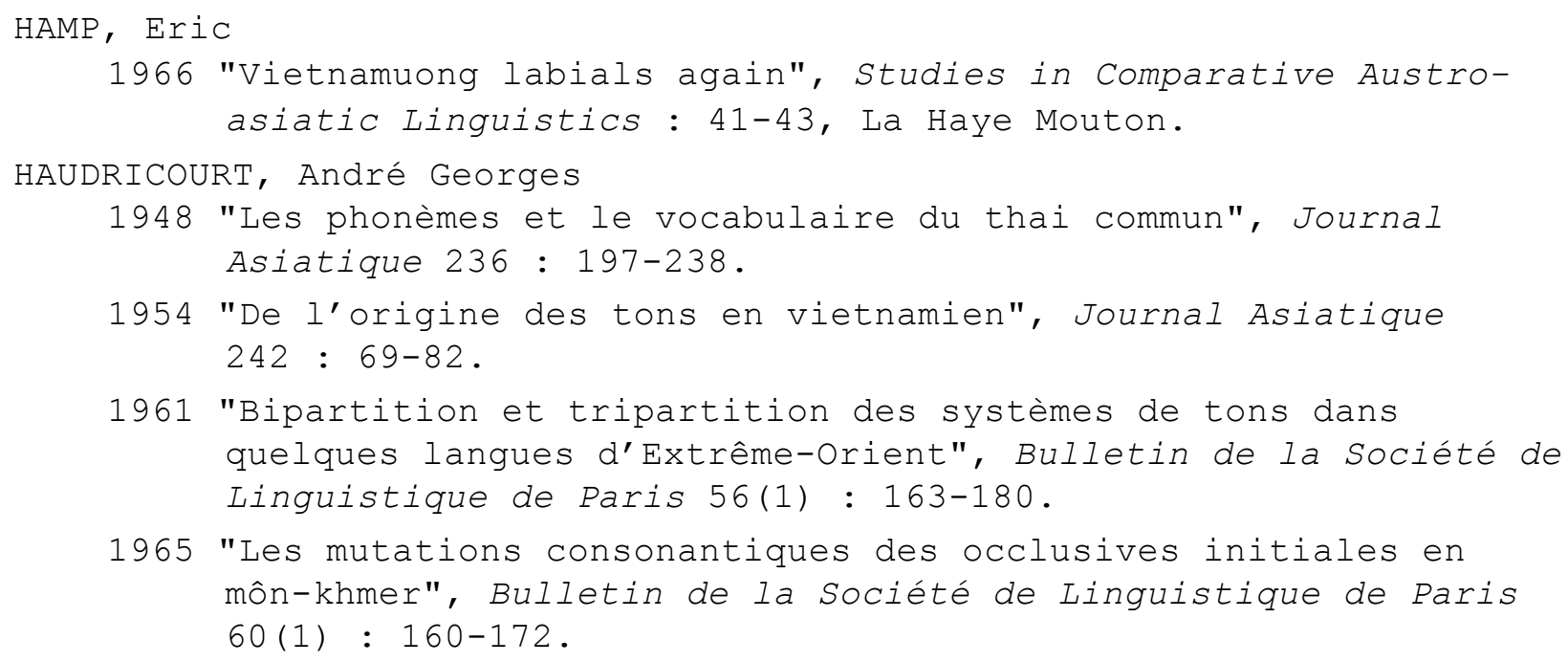

1965 "Les mutations consonantiques des occlusives initiales en môn-khmer", Bulletin de la Société de Linguistique de Paris $60(1): 160-172$.

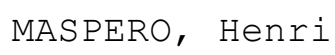

1912 "Etude sur la phonétique historique de la langue annamite : Les initiales", Bulletin de l'Ecole Française d'ExtrêmeOrient $12(1): 1-127$.

MARTINET, André

1956 La description phonologique, Paris, Genève.

1981 "Fricatives and spirants", Chatterji Commemoration Volume. The University of Burdwan, West Bengal, India. pp. 145-151.

THOMPSON, Laurence C.

1965 A Vietnamese grammar, Seattle.

1976 "Proto-viet-muong phonology", Austroasiatic Studies II : 1113-1203. Oceanic Linguistic Special Publication $n^{\circ} 13$, Hawaii.

\section{BIBLIOGRAPHIE COMPLÉMENTAIRE (titres postérieurs à 1982)}

Ferlus, Michel

1992 "Histoire abrégée de l'évolution des consonnes initiales du Vietnamien et du SinoVietnamien", Mon-Khmer Studies 20 : 111-125.

Nguyễn Tài Cẩn

1995 Giáo trình lịch sử ngữ âm tiếng Việt [Cours de phonétique historique de la langue vietnamienne]. Hànội: Nhà xuất giáo.

Nguyễn Văn Lợi

1993 Tiểng rục [La langue rục]. Hànội: Nhà xuất bản khoa học xã hội

Suwilai Premsrirat

2000 So (Thavung) preliminary Dictionary. Institute of Language and Culture for Rural Development, Mahidol University.

Vương Lộc

1995 An nam dịch ngữ. Nhà Xuất Bản Đà Nẵng. 